\title{
Connected networks of sea lice populations: dynamics and implications for control
}

\author{
Thomas P. Adams ${ }^{1, *}$, Roland Proud ${ }^{2,3,4}$, Kenneth D. Black ${ }^{1}$ \\ ${ }^{1}$ Scottish Marine Institute, Scottish Association for Marine Science, Dunbeg, Oban, Argyll PA37 1QA, UK \\ ${ }^{2}$ Pelagic Ecology Research Group, University of St. Andrews, St. Andrews KY16 8LB, UK \\ ${ }^{3}$ Australian Antarctic Division, Kingston, TAS 7050, Australia \\ ${ }^{4}$ Institute of Marine and Antarctic Studies, University of Tasmania, Hobart, TAS 7004, Australia
}

\begin{abstract}
In studies of the population dynamics of parasitic sea lice and the implications of outbreaks for salmon farms, several types of mathematical models have been implemented. Delay differential equation models describe the temporal dynamics of average adult lice densities over many farm sites. In contrast, larval transport models consider the relative densities of lice at farm sites by modelling larval movements between them but do not account for temporal dynamics or feedbacks created by reproduction. Finally, several recent studies have investigated spatiotemporal variation in site lice abundances using statistical models and distance-based proxies for connectivity. We developed a model which integrates connectivity estimates from larval transport models into the delay differential equation framework. This allows representation of sea lice developmental stages, dispersal between sites, and the impact of management actions. Even with identical external infection rates, lice abundances differ dramatically between farms over a production cycle (dependent on oceanographic conditions and resulting between-farm connectivity). Once infected, lice dynamics are dominated by site reproduction and subsequent dispersal. Lice control decreases actual lice abundances and also reduces variation in abundance between sites (within each simulation) and between simulation runs. Control at sites with the highest magnitude of incoming connections, computed directly from connectivity modelling, had the greatest impact on lice abundances across all sites. Connectivity metrics may therefore be a reasonable approximation of the effectiveness of management practices at particular sites. However, the model also provides new opportunities for investigation and prediction of lice abundances in interconnected systems with spatially varying infection and management.
\end{abstract}

KEY WORDS: Metapopulation $\cdot$ Spatial dynamics $\cdot$ Dispersal $\cdot$ Population connectivity $\cdot$ Sea lice management

\section{INTRODUCTION}

Lepeophtheirus salmonis L. is one of the most intensely studied parasitic sea lice due to its preference for commercially managed fish species such as Atlantic salmon Salmo salar L. and sea trout $S$. trutta L. In addition to impacts on fish welfare, operating costs, and logistical issues at fish farm sites (Costello 2006), interactions with and consequent impacts on wild fish populations have also received much attention (Ford \& Myers 2008, Costello 2009), with associated negative

\footnotetext{
*Corresponding author: Thomas.Adams@sams.ac.uk
}

publicity for the industry (BBC News 2012). Sea lice control takes various forms, in particular chemical treatments (either in feed or as a bath) and use of cleaner fish such as wrasse, with spatially and temporally coordinated management of farms at local and regional scales (Scotland-wide, for example; CoGP Management Group 2011). Despite these efforts, outbreaks still occur (SSPO 2014). Understanding the dynamics of outbreaks clearly has potential economic and ecological benefits, such as reduced stock loss and medicinal use, as well as reduced impact on wild fish populations.

() The authors 2015. Open Access under Creative Commons by Attribution Licence. Use, distribution and reproduction are unrestricted. Authors and original publication must be credited. 
The complexities of lice dynamics on farms result from (among other factors) their environmental context, the interactions between wild and farmed fish, and the dispersal of pelagic lice larvae. Production cycles are generally preceded by a period during which all farms in a management area are left to fallow, meaning that initial infections at farms must come from wild sources. Subsequently, reproduction at farm sites leads to an additional infection pressure via the pelagic dispersal of sea lice larvae (Johnson \& Albright 1991). Combined with difficulties in accurate quantification of wild fish movements and lice loadings, this means that the cause (or source) of outbreaks at particular farms is often not clear.

Various mathematical approaches have been investigated in attempts to understand these dynamics, including connectivity models, spatiotemporal statistical models, and delay differential equations. Several studies have considered the potential spread of larvae from farms (Amundrud \& Murray 2009, Stucchi et al. 2010, Asplin et al. 2014) and the resultant inter-farm connectivity (Adams et al. 2012). Such studies combine hydrodynamic models of particular study domains with biological particle tracking models incorporating larval development and mortality (Salama \& Rabe 2013). Such models are explicitly spatial, but they omit temporal dynamics. Several authors have also investigated lice dynamics using spatiotemporal statistical models, where site lice abundances are explained by environmental conditions, chemical treatment dates, farm biomass, and distance-based proxies for connectivity. These studies have underlined the importance of external infection pressure (Kristoffersen et al. 2013, 2014) and self-infection (Aldrin et al. 2013) in explaining juvenile lice abundances at farm sites. The possibility for positive feedback effects through high levels of self-infection has also been investigated (Krkosek et al. 2010).

Another approach uses delay differential equations to predict how the number of adult lice in a particular population develops over time based on physiological characteristics of the lice themselves (Tucker et al. 2002). Existing studies have fitted models to time series data aggregated over several sites (Revie et al. 2005, Gettinby et al. 2011), obtaining different parameter values for different subsets of sites by optimisation. This neglects the role of timing and location of wild fish interactions or differential connectivity between sites in explaining spatiotemporal variation in lice abundances. This article presents our first attempts to integrate estimates of spatially varying infection pressure and connectivity into a dynamic model, allowing a fresh viewpoint on the implications of pre- dicted temporal variability in connection strength between aquaculture sites. We investigate the difference in dynamics between population networks forced by single-pulse external infection and those driven by constant background external infection.

Previous studies have identified the role of wind direction in the spread of pelagic larvae such as sea lice (Amundrud \& Murray 2009). A study by Proud (2012) using a similar model suggested that sites demonstrating low variation in population density over randomly sampled wind conditions are important for overall robustness of the louse metapopulation. We made 2 key hypotheses. First, we predicted that sites with high louse output (measured directly from connectivity matrices; Adams et al. 2012) would be most important in creating high-density lice metapopulations in the dynamic model. Second, we predicted that connectivity outflux (sum of outward connection probabilities from each site estimated via particle tracking) would be an important determinant of the most important sites for management. We investigated the generic behaviour of the model and compared results with the simpler direct analysis of connectivity matrices. This article demonstrates how focussed management could potentially lead to dramatically reduced densities of lice at all sites.

\section{METHODS}

\section{Model overview}

We used the programming language Java to encode an individual population of sea lice behaving according to the SLiDESim model defined by Revie et al. (2005). Four key developmental stages of attached sea lice (chalimus, pre-adult, adult, and gravid female) are represented by model compartments, each with time-varying density defined by historical recruitment and delay differential equations. The model was extended to define a collection of lice subpopulations (on different fish farms), each exhibiting the same dynamic properties but operating separately. The temporal domain of the model was $700 \mathrm{~d}$, covering a typical Atlantic salmon production cycle.

In previous studies, background external infection pressure has been assumed to be constant, with a time-dependent spike used to fit particular dynamics (Gettinby et al. 2011). Our study defined infection pressure instead by a summation of the predicted reproductive output of the collection of subpopulations, scaled by predicted connection strengths between those populations (computed using the bio- 
physical model of Loch Fyne, Scotland, described by Adams et al. 2012). The fundamental model is thus a collection of $\mathrm{n}=9$ subpopulations interconnected by the distribution of larvae between the sites via connectivity matrices. A schematic overview of the model is given in Fig. 1.

\section{Subpopulation (single site) model}

The SLiDESim model (Revie et al. 2005) employed a series of coupled delay differential equations to describe the 4 attached stages of sea lice (1: chalimus, 2: pre-adult, 3: adult, 4: gravid female).

$$
\begin{aligned}
& \frac{\mathrm{d} \rho_{1}(t)}{\mathrm{d} t}=\beta(t)-\beta\left(t-t_{1}\right) \mathrm{e}^{-\mu_{1} t_{1}}-\mu_{1}(t) \rho_{1}(t) \\
& \frac{\mathrm{d} \rho_{2}(t)}{\mathrm{d} t}=\eta \beta\left(t-t_{1}\right) \mathrm{e}^{-\mu_{1} t_{1}}-\eta \beta\left(t-t_{1}-t_{2}\right) \mathrm{e}^{-\mu_{1} t_{1}-\mu_{2} t_{2}}-\mu_{2}(t) \rho_{2}(t)
\end{aligned}
$$

$\frac{\mathrm{d} \rho_{3}(t)}{\mathrm{d} t}=\eta \beta\left(t-t_{1}-t_{2}\right) \mathrm{e}^{-\mu_{1} t_{1}-\mu_{2} t_{2}}$ $-\eta \beta\left(t-t_{1}-t_{2}-t_{3}\right) \mathrm{e}^{-\mu_{1} t_{1}-\mu_{2} t_{2}-\mu_{3} t_{3}}-\mu_{3}(t) \rho_{3}(t)$

$\frac{\mathrm{d} \rho_{4}(t)}{\mathrm{d} t}=\eta \beta\left(t-t_{1}-t_{2}-t_{3}\right) \mathrm{e}^{-\mu_{1} t_{1}-\mu_{2} t_{2}-\mu_{3} t_{3}}-\mu_{4}(t) \rho_{4}(t)$

where $\rho_{j}$ is sea lice density per fish, $\tau_{j}$ is time spent in a particular stage, $\mu_{j}$ is mortality rate (n representing life cycle stages 1 to 4 ), and $\eta$ is the proportion of larvae that ultimately develop into females. $\beta(t)$ is the time-varying infection pressure (number of lice per fish) and in our combined model represents the sum of external infection from wild fish and from connected fish farms (including self-infection; see 'Combined model' below). In Revie's (and subsequent related, e.g. Gettinby et al. 2011) study, these parameters were estimated by fitting model output to time series of lice densities averaged over many sites.

For simplicity, it is assumed that water temperature (and/or its consequent effect on population rates) does not vary over the course of a production cycle. Sea lice larval development times are likely to be fairly insensitive to the temperature range $\left(8\right.$ to $\left.14^{\circ} \mathrm{C}\right)$ observed in Scottish waters (Stien et al. 2005).

\section{Connectivity model}

The model of Adams et al. (2012) tracked the trajectories of passive sea lice larva particles driven by modelled currents. An initial non-infective nauplii stage of approximately $4 \mathrm{~d}$ and a maximum dispersal duration of $14 \mathrm{~d}$ allowed us to compute the probability of successful dispersal between each possible pair

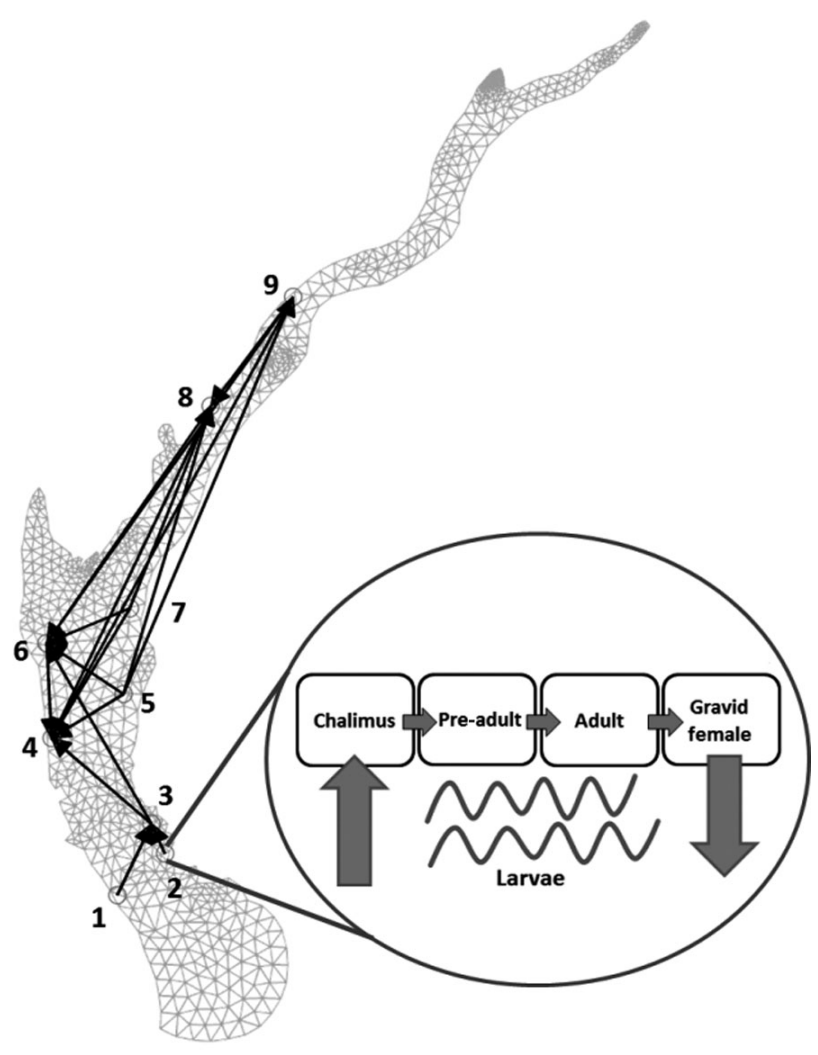

Fig. 1. Model schematic. The basic model was a network of 9 subpopulations of sea lice, each obeying the dynamic equations described by Revie et al. (2005) (see Eq. 1), connected by matrices defining inter-site dispersal probabilities. The locations of the sites are overlaid here on the hydrodynamic model mesh used to compute dispersal probabilities within Loch Fyne, Scotland (see Adams et al. 2012). Connections between sites in this diagram are indicative examples; 81 different connections (including self-connections) are possible in practice

of the $\mathrm{n}=9$ sites within Loch Fyne on the west coast of Scotland (giving a total of 81 connection probabilities, as connections are non-commutative). These were arranged in a connectivity matrix, $\boldsymbol{C}(\mathrm{n} \times \mathrm{n}$ matrix with self-infection probability on the leading diagonal; Fig. 2). We also computed the mean dispersal duration for all possible connections (another $\mathrm{n} \times$ n matrix, $\tau_{\text {; }}$ range $=4$ to $12 \mathrm{~d}$ ).

Connectivity matrices were summarised in various ways. In particular, the average probability of successful dispersal was given by summing all values in the matrix and dividing by the number of sites, i.e. $\sum_{i=1}^{\mathrm{n}} \sum_{j=1}^{\mathrm{n}} C_{i j} / \mathrm{n}$, where $i$ denotes source and $j$ denotes destination site. The outflux of each site (probability of successful dispersal from that site to any site including itself) was given by the row sums of the matrix, that is Outflux ${ }_{i}=\sum_{j=1}^{\mathrm{n}} C_{i j}$. Similarly, the influx of each site (relative number of arrivals at each site, on the assumption of equal output) was given by the matrix column sums, Influx $_{j}=\sum_{i=1}^{\mathrm{n}} C_{i j}$. 
Adams et al. (2012) performed 12 separate 14 d dispersal simulations, forced using wind data collected over 1 yr (April 2011 to March 2012), generating 12 matrices representing variation in connectivity during the year. Throughout, we refer to outflux and influx averaged over all 12 matrices as connectivity outflux and connectivity influx. During this period, the wind conditions were predominantly southwesterly (directed towards the northeast, as is usually experienced in this region; up-loch or landward), opposing the generally seaward transport of surface water layers. In reality, salinity avoidance by dispersing nauplii-stage lice could affect the extent to which surface transport dominates lice dispersal patterns and allow enhanced up-loch movement of lice larvae (Costello 2009).

\section{Combined model}

A combination of the 2 models described above was simulated over a full production cycle $(22 \mathrm{mo})$. At time $t$, the reproductive output of larvae from a particular site was defined as

$$
F_{i}(t)=\rho_{4, i}(t) N_{i}(t) f
$$

where $N_{i}(t)$ is the number of fish at site $i$ at time $t$, and $f$ is the number of larvae successfully produced per

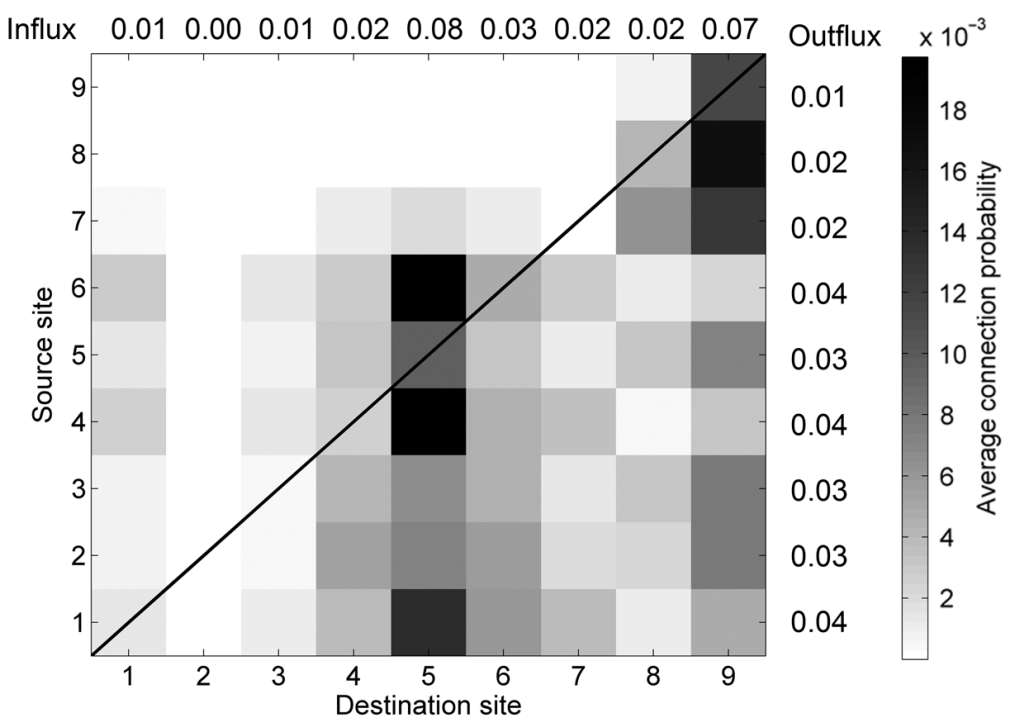

Fig. 2. Average connectivity matrix for 9 sites in Loch Fyne, from twelve 2 wk simulations using wind-forcing data from throughout the year. Darker colour indicates higher connection probability (probability of successful dispersal from one site to another). Connections indicate predominantly northeasterly transport within the loch, due to prevailing southwest wind forcing of surface layers. Column and row sums are the connectivity influx and outflux, respectively, of each model site unit time (1 d) by each female. The number of larvae arriving (per fish) at site $i$ at time $t$ was a combination of the sum over all such outputs and the background infection pressure:

$\beta_{i}(t)=$ Pulse external + Constant external + internal dispersal

$=\frac{1}{N_{i}(t)}\left(\alpha_{1} l\left(t=t_{\mathrm{Ext}}\right)+\alpha_{2} H\left(t-t_{\mathrm{Ext}}\right)+\sum_{j=1}^{9} C_{j i} F_{j}\left(t-\tau_{j i}\right)\right)$

and $I$ is the indicator function ( 1 for $t=t_{\text {Ext, }} 0$ otherwise). $\alpha_{2}$ is the ongoing external infection pressure, and $H$ is the Heaviside step function ( 0 for $t<t_{\mathrm{Ext}}$ and 1 otherwise; background infection only occurred after a certain time at which infected wild salmon were assumed to pass in the vicinity of the farms). We assumed that a production cycle began in November (i.e. using $\mathrm{S}^{1} \frac{1}{2}$ smolts, i.e. produced in 6 mo from hatching), and $t_{\mathrm{Ext}}$ was set to coincide with the spring run of wild salmon. Sea lice levels are typically low during this early part of the production cycle in Scotland. We considered spatially homogeneous (all sites) and inhomogeneous (individual sites) external infections (see 'Scenarios and investigations' below).

$C_{j i}$ is the probability of dispersal from site $i$ to site $j$ (incorporating mortality during dispersal; see Adams et al. 2012). $\tau_{j i}$ is the mean dispersal time from site $j$ to site $i$. Our baseline simulations fixed inter-site dispersal duration to $\tau_{j i}=14 \mathrm{~d}$ for all site pairs. To represent temporal variation in site connectivity, a new connectivity matrix $\boldsymbol{C}$ was selected at random from the 12 available matrices once every $30 \mathrm{~d}$. We tested model sensitivity to variation in this reselection interval, which essentially parameterises persistence of particular current patterns.

\section{Parameterisation}

We used several parameter values following those obtained by previous studies (Revie et al. 2005, Stien et al. 2005, Gettinby et al. 2011), as these adequately reflect expected development times under local environmental conditions (Stien et al. 2005). We did not attempt to model a specific empirical population but instead investigated scenarios highlighting generic aspects of model behaviour. The timestep of the model was $1 \mathrm{~d}$. All model parameters (approximating L. salmonis; hereafter referred to as 'lice') are summarised in Table 1. 
Table 1. Model parameters. Life stage duration parameters of salmon louse Lepeophtheirus salmonis follow those of Revie et al. (2005) and approximate values estimated by Stien et al. (2005) at 10 to $11^{\circ} \mathrm{C}$

\begin{tabular}{|c|c|c|c|c|}
\hline Paramete & Description & Value & Unit & Source \\
\hline$t_{1}$ & Chalimus stage duration & 15 & d & Revie et al. (2005) \\
\hline$t_{2}$ & Pre-adult stage duration & 10 & d & Revie et al. (2005) \\
\hline$t_{3}$ & Adult stage duration & 20 & $\mathrm{~d}$ & Revie et al. (2005) \\
\hline$t_{4}$ & Gravid female stage duration & 37 & d & $\begin{array}{l}\text { Johnson \& Albright (1991), } \\
\text { Heuch et al. (2000) }\end{array}$ \\
\hline$t_{\text {Ext }}$ & Time range of initial pulse infection & $154-174$ & $\mathrm{~d}$ & Revie et al. (2005) \\
\hline$\tau_{j i}$ & Larval duration & 14 & d & Amundrud \& Murray (2009) \\
\hline$\mu_{1}$ & Chalimus mortality rate & 0.0255 & $d^{-1}$ & Revie et al. (2005) \\
\hline$\mu_{2}$ & Pre-adult mortality rate & 0 & $d^{-1}$ & Revie et al. (2005) \\
\hline$\mu_{3}$ & Adult mortality rate & 0 & $d^{-1}$ & Revie et al. (2005) \\
\hline$\mu_{4}$ & Gravid female mortality rate & 0.0269 & $\mathrm{~d}^{-1}$ & $1 / t_{4}$ \\
\hline$f$ & $\begin{array}{l}\text { Number of eggs per day per gravid female that reach } \\
\text { chalimus stage }\end{array}$ & $2,5,10$ & lice $\mathrm{d}^{-1}$ & $\begin{array}{l}\text { Johnson \& Albright (1991), } \\
\text { Heuch et al. (2000) }\end{array}$ \\
\hline$\alpha_{1}$ & Background infection of chalimus per fish (initial pulse) & $0,0.01,0.1,1$ & lice fish $^{-1} \mathrm{~d}^{-1}$ & Revie et al. (2005) \\
\hline$\alpha_{2}$ & Background infection of chalimus per fish (constant) & $0,0.01,0.1,1$ & lice $f_{i s h}{ }^{-1} \mathrm{~d}^{-1}$ & Gettinby et al. (2011) \\
\hline
\end{tabular}

\section{Management treatments}

The impact of simulated chemical treatments that kill lice living on fish at farm sites was investigated. Two forms of treatment in common use were simulated here. First, a bath treatment known as Alphamax (active ingredient deltamethrin) may be used to directly kill lice living on the skin of fish. A treatment event was simulated by an instantaneous one-off reduction in density of all stages of lice of $95 \%$ (following Gettinby et al. 2011), though this may be a substantial overestimate of efficacy for certain contexts (Jimenez et al. 2013). We assumed that there was no residual effect.

Second, an in-feed treatment known as SLICE (active ingredient emamectin benzoate) may be used; treatment takes effect immediately and causes a decline in lice numbers for around $28 \mathrm{~d}$, followed by gradual recovery. This was simulated in the model by a daily reduction in density of all stages of lice (at farm sites) of $17.4 \%$, for $28 \mathrm{~d}$, matching the exponential decline implemented by Gettinby et al. (2011) (reaching almost zero lice at $28 \mathrm{~d}$, though again this is an overestimate of efficacy in comparison with controlled trials; MSD Animal Health 2012). After this point, we allowed simulated lice numbers to recover unhindered.

For simulations including management, all managed sites followed an identical treatment pattern based on that used at the real sites. SLICE treatments took place on Days 180, 330, and 450, and Alphamax treatment took place on Days 540 and 570 .

\section{Scenarios and investigations}

Sensitivity of a single population model behaviour to mortality and reproduction parameters has been investigated previously (Revie et al. 2005). Our model incorporated multiple sites and the additional factor of inter-site dispersal. Simulations assumed that all 9 sites in the loch network were stocked with the same number of fish, that this number was fixed for the duration of the production cycle, and that fish did not grow (that is, carrying capacity is fixed over time; an assumption implicit in Revie et al.'s study). This simplification makes comprehension of model results more straightforward, though precise stocking data could easily be incorporated into the model for specific predictive purposes. For each scenario/ parameter set, 100 repeat runs were performed.

A first suite of simulations investigated variation in intensity of the pulse infection $\alpha_{1}[0,0.01,0.1,1]$, constant infection $\alpha_{2}[0,0.01,0.1,1]$, and louse egg production rate $f[2,5,10]$, considering how external infections $\left(\alpha_{1} \neq 0\right.$ or $\left.\alpha_{2} \neq 0\right)$ at individual sites, subsets of the sites, and all sites affected lice metapopulation dynamics. These simulations did not include chemical treatments, and lice numbers at the sites were allowed to develop unhindered. Comparison of these results with connectivity influx and outflux of the sites was made to determine the extent of insights gained without recourse to the complete dynamic population model.

We also investigated the impact of management events on system dynamics, with the goal of assess- 
ing the relative importance (or indeed necessity) of chemical treatment at particular sites. For these scenarios, we applied the full cycle treatment schedule outlined above to each site in turn, subsets of 3 , and all sites together over the full range of $\alpha_{1}, \alpha_{2}$, and $f$. For these management runs, we assumed that external infection pressures $\alpha_{1}$ and $\alpha_{2}$ were identical at all sites.

Finally, we investigated the effects of altering the connectivity matrix reselection interval noted in 'Combined model' (from $1 \mathrm{~d}$ up to full cycle, altering the level of environmental variation in each simulation run) and the impact of using estimated pairwise dispersal times (matrix $\tau$ ) in place of the fixed $14 \mathrm{~d}$ dispersal duration.

\section{RESULTS}

\section{Basic model behaviour-external infection scenarios}

The initial condition was zero lice at each site. In all cases, lice first arrived from the date of first external infection (as defined by the parameters $\alpha_{1}$ and $\alpha_{2}$ in Eq. 3). Following this, lice numbers increased due to both external and between-site infections. Fig. 3 shows total lice numbers at all 9 model sites forced by constant background external infection at all sites.

In spite of the evenly spread external infection, some sites had much higher densities than others. Some sites also experienced much greater variation
(1)

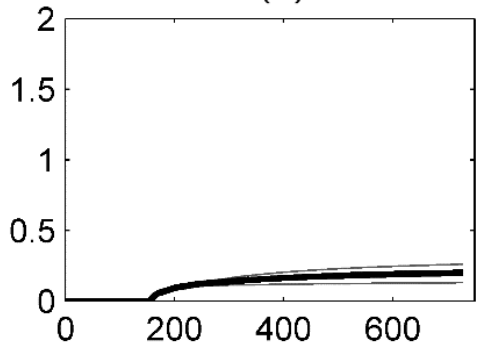

(4)

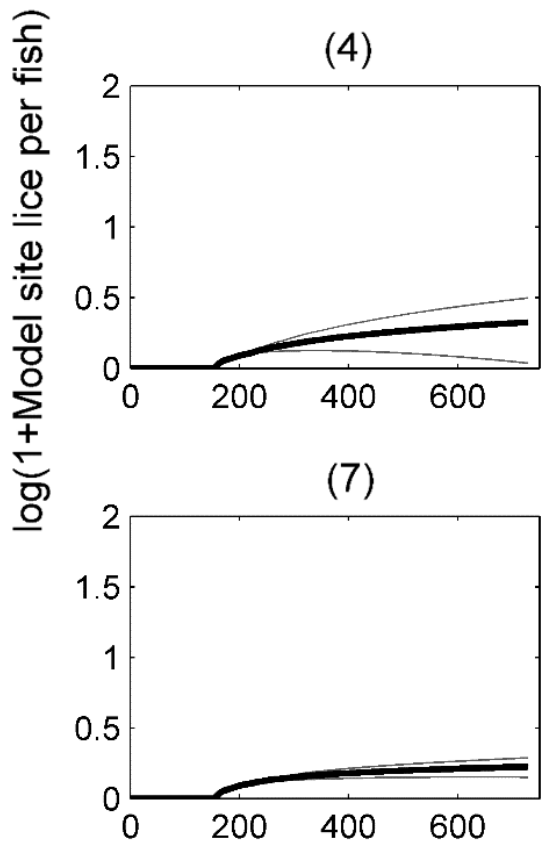

(2)

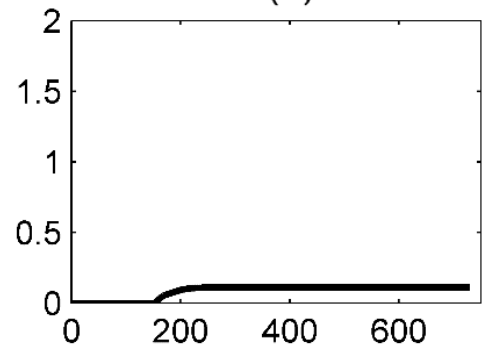

(5)

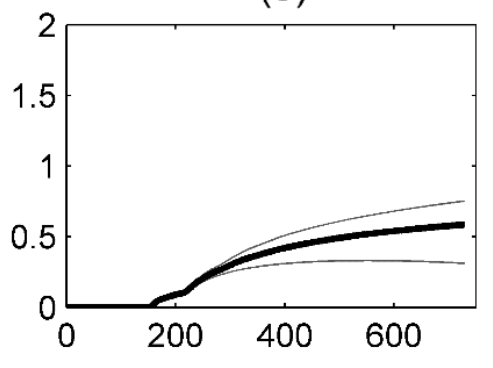

(8)

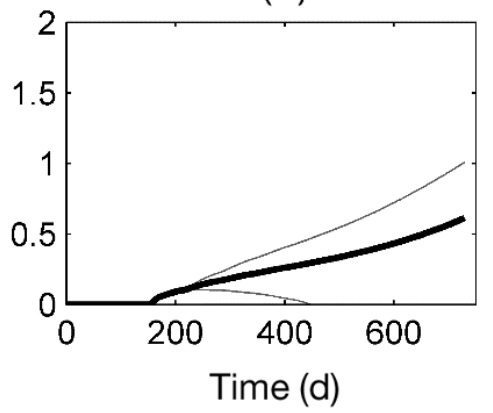

(3)

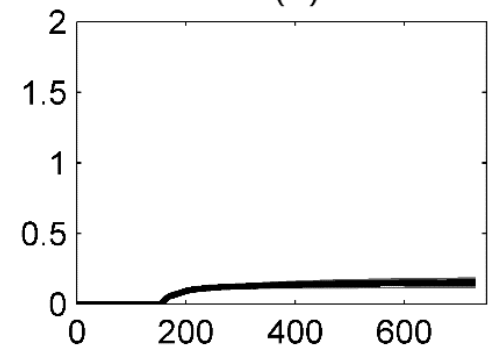

(6)

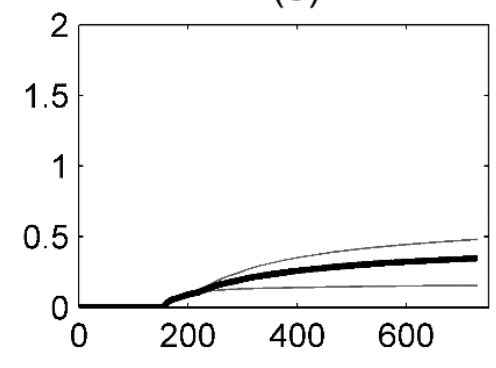

(9)

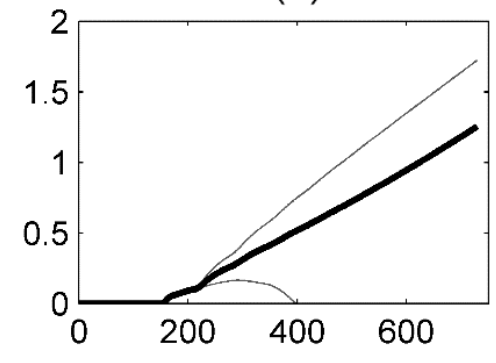

Fig. 3. Mean lice density (thick black line) and standard deviation over 100 runs (grey lines) of lice at each site (1 to 9), plotted over the duration of a production cycle, assuming a constant external infection of 0.01 lice fish ${ }^{-1} \mathrm{~d}^{-1}$, reproduction of 5 lice adult female ${ }^{-1} \mathrm{~d}^{-1}$ at all sites, and no lice reduction management events. Numbers do not increase exponentially at all sites. Rather, they may reach a plateau where mortality and infection (which, due to connectivity, is different for each site) are in balance. At approximately realistic parameter values, dynamics are governed by on-farm reproduction, meaning that dynamics are essentially identical in the pulse infection scenario 
(a)

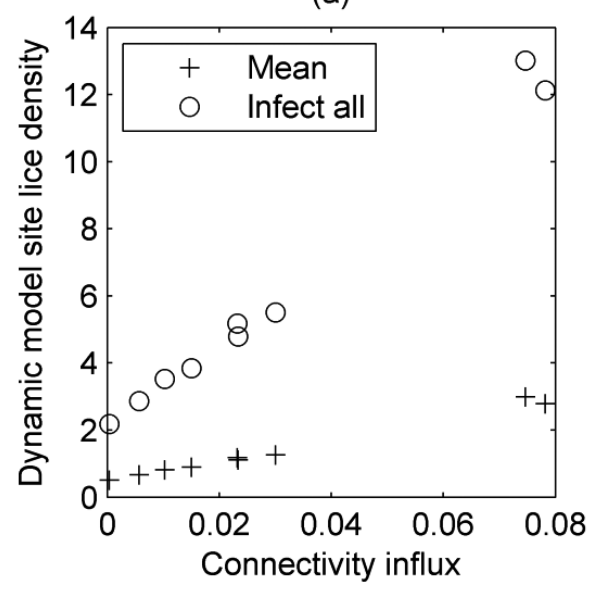

(b)

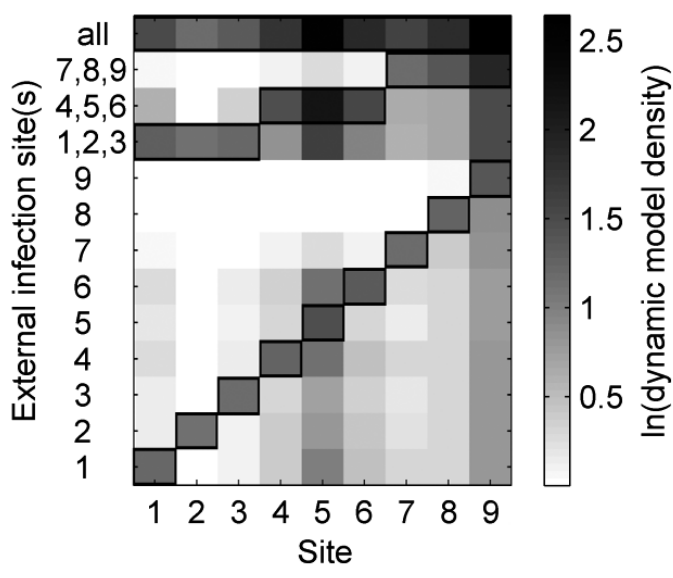

Fig. 4. Individual site densities. (a) Average site connectivity influx (over all 12 connectivity matrices) versus model mean site lice density for the pulse infection scenario, with external infection of 0.01 lice fish ${ }^{-1} \mathrm{~d}^{-1}$ and reproduction of 5 lice adult female $\mathrm{e}^{-1} \mathrm{~d}^{-1}$. The mean of all individual site pulse infection scenarios (that is, external infection at different sites) is shown by ' + ', while the case where all sites receive external infection is indicated by ' $O$ '. (b) Mean lice density at each site (over a whole model run) for runs where only particular sites receive external infection. Each row corresponds to an infection scenario, with the externally infected site outlined for clarity. In this case, connectivity influx is a weaker predictor of combined model density. Note the tendency for northward spread of lice between the sites

in lice density between runs than others. Differences in model site lice densities are due to uneven connectivity between locations, caused by spatial variation in water movements. When external infection occurred equally at all sites (and when taking mean site densities over all scenarios where only single sites were infected externally; Fig. 4a), connectivity influx was a very strong predictor of each site's mean louse density in the combined model, regardless of whether external infection was a short pulse or constant. Relative density between sites was not affected by whether external infection was a pulse or constant.

In cases where a single site (or a subset of sites) received external infection, this relationship broke down; connectivity influx no longer predicted individual site lice density. In these cases, the site which received external infection always had higher louse densities in the combined model than all other sites. If these sites were omitted from analysis, there was a weak correspondence between connectivity influx and combined model site louse density (not shown), most notably when sites close to the mouth of the loch were the sole infection point. In general, external infection at particular sites resulted in higher predicted lice densities at downstream sites (those with strong incoming connections from the externally infected sites; Fig. 4b).

The site of external infection affects mean louse density over the whole network. Average density over all sites in the loch decreased when the externally infected site was more northerly (from Sites 1 to 9; Fig. 5a). Mean model lice density over all sites and the full cycle was almost exactly linearly related to the connectivity outflux of the externally infected site(s), whether this was a single site or a combination (Fig. 5b). This relationship held for pulse and constant infection scenarios. Fig. 5 also demonstrates that at this (approximately realistic) reproduction level, mean lice density was not increased by ongoing external infection versus pulse infection; system dynamics were dominated by reproduction in louse subpopulations on the farms and subsequent intersite dispersal.

Altering the balance between pulse infection, continuous infection, and reproduction rate did not affect the magnitude of individual site lice densities relative to one another (assuming that the externally infected sites do not change; not shown). Reselecting the connectivity matrix more frequently reduces variation between simulation runs; runs became more average. If connectivity was fixed for the duration of the production cycle, individual site dynamics became more directly predictable from the connectivity matrices. For sites with the highest connectivity influx, variation between runs increased exponentially over time (contrasting with the matrix reselection case, where variable connectivity had a mediating effect on variation in louse abundance; not shown). Replacing the fixed dispersal duration with estimated mean pairwise inter-site dispersal times increased lice abundances slightly but did not fundamentally alter behaviour (not shown). 
(a)

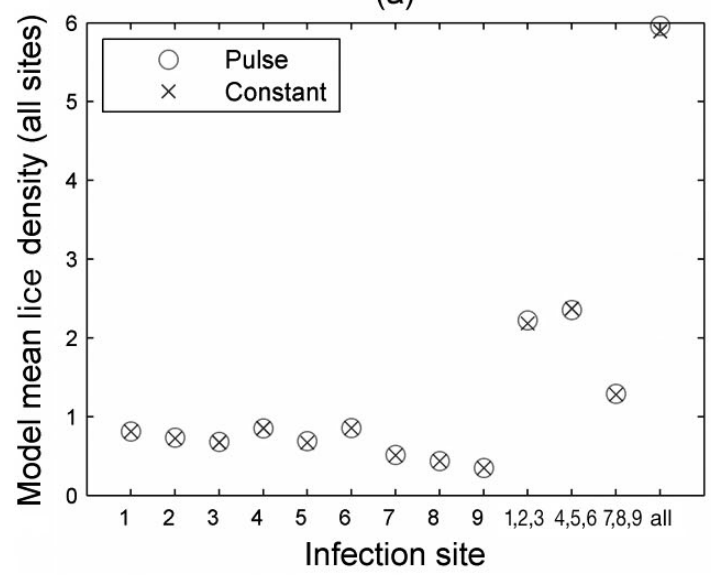

(b)

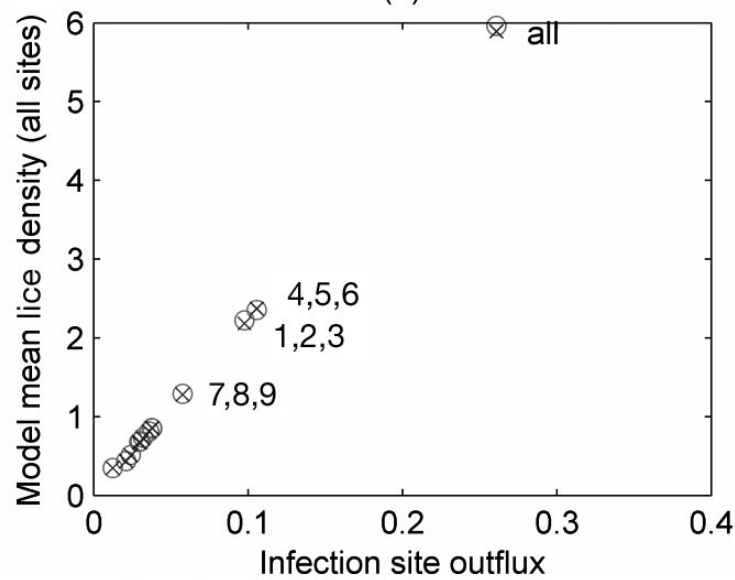

Fig. 5. Mean loch densities. (a) Changing source site. Mean lice density across all 9 sites when particular sites receive external infection. (b) Connectivity outflux versus model mean site density over time. Unlabelled points represent external infection at individual sites, while combinations of sites are labelled. Values for the pulse scenarios $\left(20 \mathrm{~d}\right.$ at 0.1 lice fish $\left.{ }^{-1} \mathrm{~d}^{-1}\right)$ are denoted by ' $\bigcirc$ ', and constant scenarios $\left(0.1\right.$ lice fish ${ }^{-1} \mathrm{~d}^{-1}$ throughout) are denoted by ' $\otimes$ '

(1)

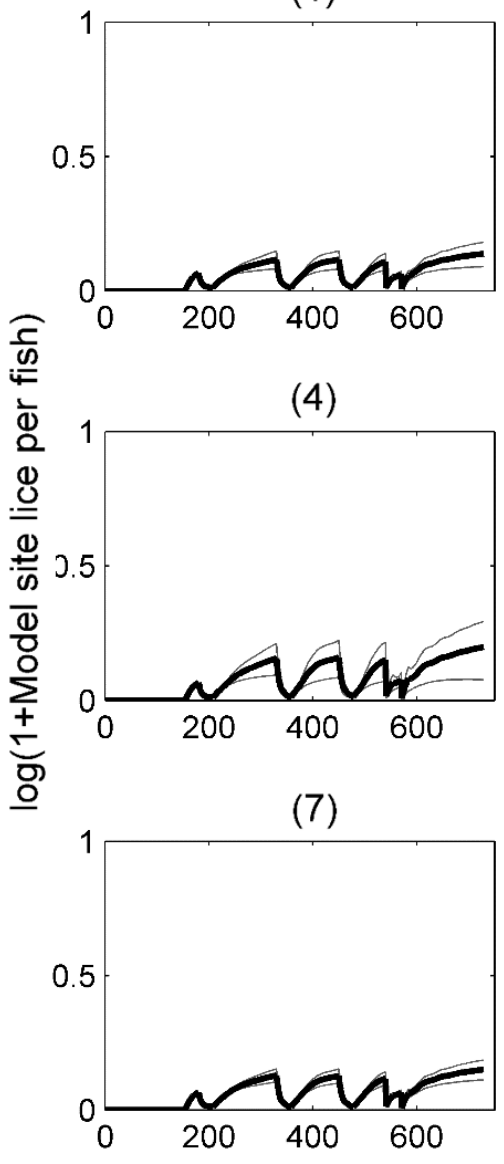

(2)

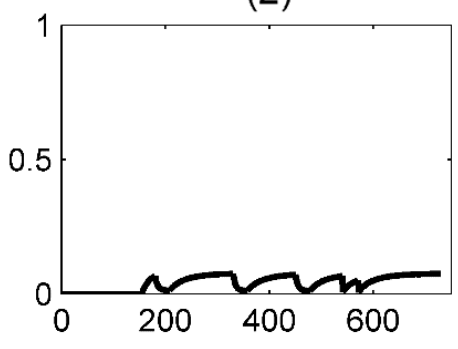

(5)

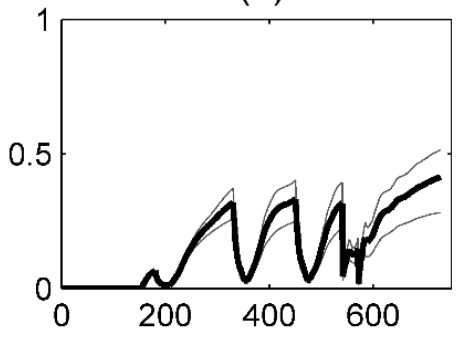

(8)

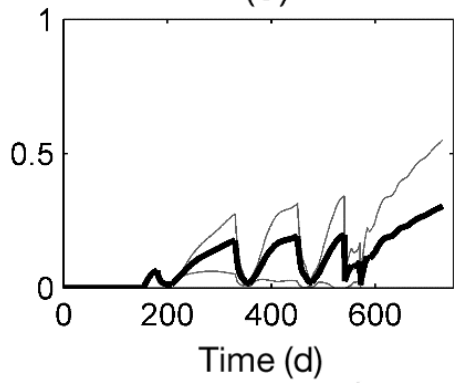

(3)

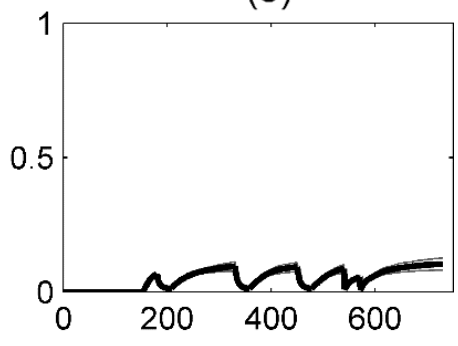

(6)

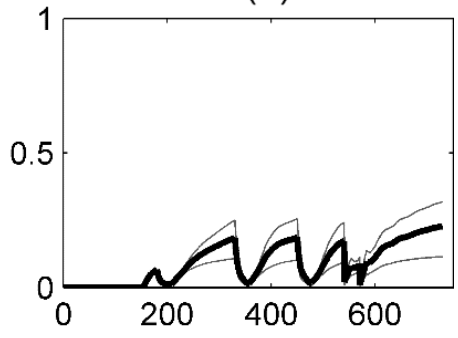

(9)

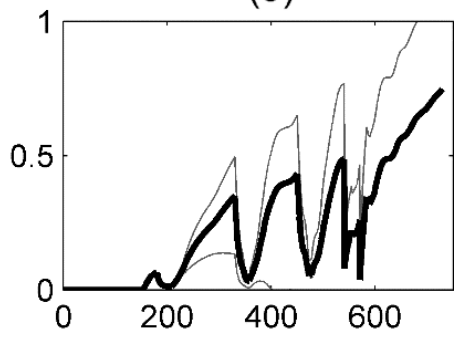

Fig. 6. Lice density at each model site over the duration of the production cycle, when all sites were managed according to the schedule presented in 'Methods: Management treatments' (each site receives constant external lice infection of 0.01 lice fish ${ }^{-1}$ $\mathrm{d}^{-1}$ and reproduction of 5 lice adult female ${ }^{-1} \mathrm{~d}^{-1}$; same parameters as Fig. 3) 
(a)

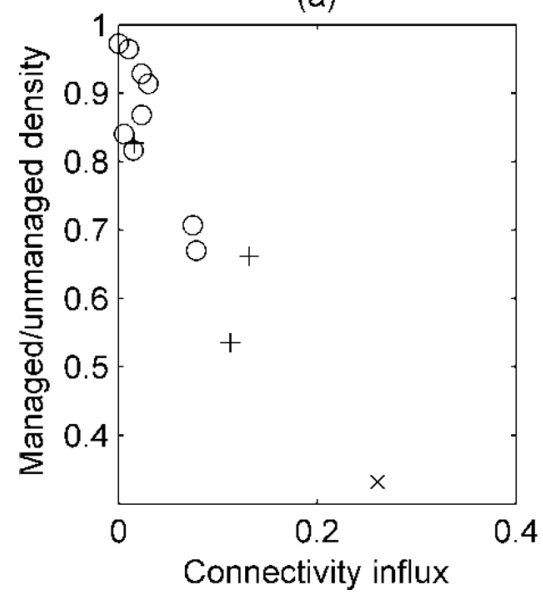

(b)

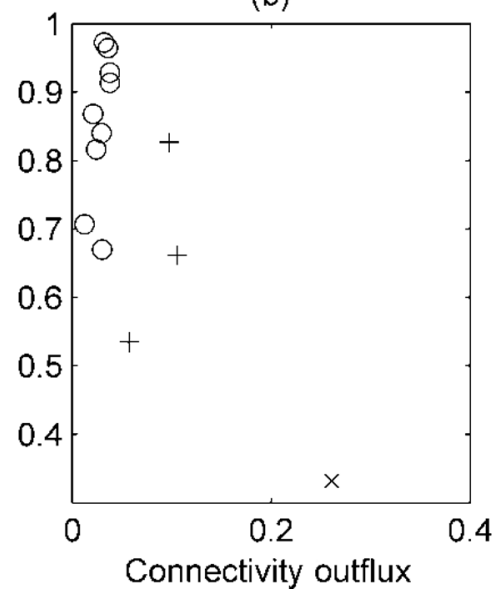

(c)

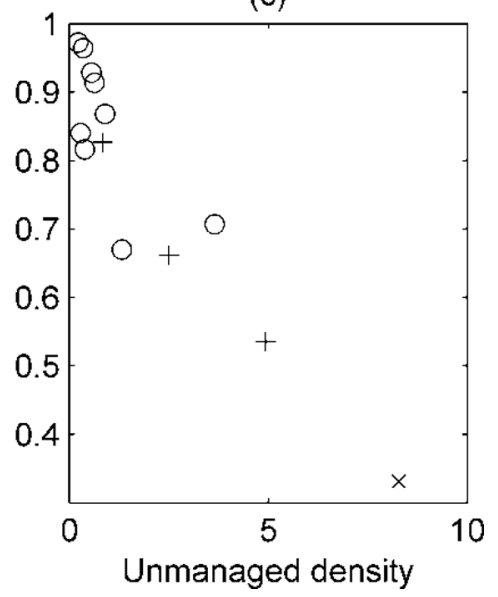

Fig. 7. Impact of applying management protocol to model sites, measured by proportional mean lice abundance over all model sites over the whole production cycle, relative to the unmanaged case. Again, parameters applied were a constant external infection of 0.01 lice fish ${ }^{-1} \mathrm{~d}^{-1}$ and reproduction of 5 lice adult female $\mathrm{d}^{-1}$. Impact of managing particular sites is plotted versus (a) connectivity influx, (b) connectivity outflux, and (c) unmanaged density of those sites. Individual sites are indicated by ' $O$ ', groups of 3 sites by ' + ', and all sites managed together by ' $x$ '

\section{Effect of chemical treatment}

Chemical treatments were simulated in the model via the direct removal of lice from the subpopulation sites, reducing the density of lice present at the sites (Fig. 6) by between 4 and $67 \%$, depending on which sites were treated (Fig. 7). Management also homogenised simulation runs; each management event brought lice density to a similar low level in each run, reducing the variation in lice density.

A common goal of management may be to achieve maximum effect with minimum cost or effort. We therefore assessed the relationship between site connectivity metrics with the impact of focussing management on those particular sites (individual sites and groups of sites $[1,2,3],[4,5,6],[7,8,9])$. Connectivity influx of the managed site(s) correlated strongly and positively with the resulting reduction in mean (whole system, whole cycle) population model density (binomial generalised linear model proportion of deviance explained, $D_{p}=0.70$, at default parameter values; Fig. 7a). The correlation between site connectivity outflux and mean density reduction was poor $\left(D_{p}=0.08\right)$, and the sign of the relationship changed depending on whether groups of sites were included in the regression. This is surprising, as intuitively, highly outwardly connected sites might be expected to be the most important in producing high louse numbers across the system. Unmanaged density of a site was also a good predictor of the impact of managing it $\left(D_{p}=0.63\right)$, but we observed no link with inter-run variability in lice densities at a particular site. These patterns held for all pulse and constant infection scenarios considered.

As noted previously, connectivity influx was also correlated with population model lice density. It is therefore possible that the reduction in lice abundances was due to management focussed on high influx sites. With this in mind, we performed a similar analysis using mean abundances, omitting the sites at which management was focussed. In this case, site connectivity influx remained a better predictor of management impact, but both were only weakly correlated ( $D_{p}$ explained by connectivity influx and outflux were 0.14 and 0.09 , respectively), while unmanaged site density became almost completely uncorrelated $\left(D_{p}=0.0008\right)$.

\section{DISCUSSION}

By combining 2 established approaches (connectivity modelling and dynamic life cycle population modelling), we have gained several insights into the contribution of different processes affecting the population dynamics of sea lice at individual aquaculture sites. At realistic parameter values, model behaviour was dominated by reproduction on (and connectivity between) sites, meaning that there was no substantial difference in lice densities between pulse and constant external infection cases. If this translates to reality, this adds support to the idea that inappropriately managed farms pose a significant threat by increasing the densities of lice both at farm sites and moving freely in the water column. 
Variation in connectivity led to rather different lice abundances between sites, even in the case of equal external infection rate, and spatial directionality in the connectivity matrix was evident in the combined model results. Lice control measures were effective at reducing lice abundances, achieving 4 to $67 \%$ reduction in mean lice abundance over a production cycle. Lice abundance at sites was generally proportional to the weight of incoming connections, as was the impact of managing (treating to remove lice) at particular sites in isolation. In this system, dispersal patterns were predominantly northward, despite the tendency for surface freshwater layers to flow in a seaward direction. In reality, juvenile lice may experience enhanced inshore transport by moving more deeply during early stages of development (Costello 2009). Our model used a single near-surface vertical position, but stage-dependent and salinity-driven larval migration, mortality, or settlement success (Bricknell et al. 2006) could easily be incorporated into the underlying connectivity model.

We found that management was most effective (in terms of reducing average lice densities over the whole loch and production cycle) when focussed on model sites with high connectivity influx or sites with high unmanaged density (under the assumption that external infection at all sites is equal). This contrasts with the more intuitive assumption that lice control at high connectivity outflux sites would be most important. Interestingly (and in contrast to the previous study by Proud 2012), variability in combined model density at a specific site over randomly selected wind scenarios was not a useful indicator of the impact of managing those specific sites in isolation. Disentangling mean loch lice reductions from the density reduction at the focal managed site was not straightforward, as correlations were greatly reduced when omitting this from full loch averages. Using the current system as an example, it is therefore difficult to deduce the extent to which management treatment affects spread through the loch, though this does not alter the main result: that management at high density (or high connectivity influx) sites is likely to offer the most cost-effective reduction in lice numbers.

The present study builds on the previous work of other authors (Revie et al. 2005, Gettinby et al. 2011), adding an explicit representation of spatially varying transmission and external infection. This allowed us to investigate how spatially varying dispersal and infection create differential lice abundances at farm sites within the same loch and to investigate the impact of spatially varying management schemes, rather than considering only average behaviour over many farms. In certain scenarios, metrics computed directly from the connectivity matrices are likely to provide a good surrogate for dynamic population modelling (equality of sites, equality of infection, or when considering the average behaviour of many different site groups or time periods). Indeed, in the cases where all sites receive equal levels of external infection and at accepted levels of sea louse reproduction, between-site dispersal and consequent crossinfection rapidly dominate dynamics over external infection. In such cases, studies of baseline connectivity between sites (Adams et al. 2012, Salama \& Rabe 2013) may be sufficient.

In reality, external infection may be less homogeneous. Not all river catchments in Loch Fyne are home to salmonids (Argyll Fisheries Trust 2010), and proximity to those that are (and relevant migration routes or particular hydrodynamic conditions in their localities) may alter the level of external infection pressure that sites face. Middlemas et al. (2013) found a link between sea lice abundances on wild Scottish sea trout and habitat proximity to farms, and we see no reason that the reverse (that external infection pressure at farm sites should increase with proximity to wild fish populations) should not be true. In conjunction with the inhomogeneity (and asymmetry) of inter-site connections, inhomogeneous external infection may mean that in reality some sites have dynamics governed by external infection, while others are governed by between-site dispersal. Empirical information is therefore likely to be invaluable in the configuration of the combined model, should one wish to predict spatiotemporal variation in lice numbers in a real system. Given such information, our combined model would allow a more considered (and more efficient and lower impact) approach to lice management to be applied.

The combined model presented here is based on a foundation composed of hydrodynamic modelling and biological particle tracking and necessarily assumes the inherent strengths, weaknesses, and assumptions of these approaches, in addition to the assumptions that are made directly in its formulation (though these do not affect our basic results). For prediction of sea lice abundances over time, simpler statistical approaches directly dealing with empirical data may be more effective (e.g. Aldrin et al. 2013, Rogers et al. 2013, Kristoffersen et al. 2014) and allow for the integration of a broad range of influencing factors. However, by building up from the physiological properties of sea lice, our approach allows a more fundamental look at how external infections, on-site reproduction, inter-site connectivity, and manage- 
ment activities affect lice abundances in connected networks. In common with statistical studies, our study underlines the importance of accounting for connectivity when considering variation in lice abundances in a spatial context. But we are also able to provide insights on management: coordinated management at many sites is clearly ideal, but focussing on the highest receiving sites (calculated from the connectivity matrix) is a good second choice.

Our study did not investigate the potential impacts of cleaner fish on lice densities. Co-stocking of salmon with wrasse species has been found in the correct conditions to be a highly effective approach to reducing lice numbers, particularly mature stages (Skiftesvik et al. 2013). However, finely tuned relative stocking densities are essential to ensure the welfare of both wrasse and salmon. Furthermore, ensuring that lice remain the preferred food choice of the wrasse depends on maintaining minimal biofouling of infrastructure and the amount of additional food provided for them. If such issues (and others relating to supply; MCS 2013) are dealt with, wrasse have the potential to almost completely prevent the development of reproducing sea lice in salmon cages. Applied at sites where high levels of external infection exist, or sites with high connectivity influx, this could be more effective than chemical treatments.

Other generic questions may be investigated using the modelling approach presented here. For example, given accurate data on treatment efficacy and effect duration, the model provides an opportunity to design and optimise treatment regimes (potentially over both time and sites), reducing costs and environmental impacts of farm management. The impact of abundance thresholds and targets for adaptive management schemes could be assessed. Finally, the emergence of chemical-resistant lice (Jones et al. 2013), which has important implications for management protocol, is a pressing topic for investigation.

Acknowledgements. This work was supported by a grant from the European Fisheries Fund (European Union). We are grateful for the comments of the editor and anonymous referees that greatly improved the manuscript.

\section{LITERATURE CITED}

Adams TP, Black K, MacIntyre C, MacIntyre I, Dean R (2012) Connectivity modelling and network analysis of sea lice infection in Loch Fyne, west coast of Scotland. Aquacult Environ Interact 3:51-63

Aldrin M, Storvik B, Kristoffersen AB, Jansen PA (2013) Space-time modelling of the spread of salmon lice between and within Norwegian marine salmon farms. PLoS
ONE 8:e64039

Amundrud TL, Murray AG (2009) Modelling sea lice dispersion under varying environmental forcing in a Scottish sea loch. J Fish Dis 32:27-44

Argyll Fisheries Trust (2010) South Argyll Rivers Project, final report: survey of fish populations and habitats 200810. Argyll Fisheries Trust, Inveraray

Asplin L, Johnsen IA, Sandvik AD, Albretsen J, Sundfjord V, Aure J, Boxaspen KK (2014) Dispersion of salmon lice in the Hardangerfjord. Mar Biol Res 10:216-225

BBC News (2012) Sea lice killing 'large numbers' of salmon. http://www.bbc.com/news/uk-scotland-highlands-islands20236291 (accessed 10 Oct 2014)

Bricknell IR, Dalesman SJ, O'Shea B, Pert CC, Mordue Luntz AJ (2006) Effect of environmental salinity on sea lice Lepeophtheirus salmonis settlement success. Dis Aquat Org 71:201-212

CoGP (Code of Good Practice) Management Group (2011) A code of good practice for Scottish finfish aquaculture. http://thecodeofgoodpractice.co.uk/ (accessed 10 Oct 2014)

> Costello MJ (2006) Ecology of sea lice parasitic on farmed and wild fish. Trends Parasitol 22:475-483

Costello MJ (2009) How sea lice from salmon farms may cause wild salmonid declines in Europe and North America and be a threat to fishes elsewhere. Proc R Soc B 276: 3385-3394

Ford JS, Myers RA (2008) A global assessment of salmon aquaculture impacts on wild salmonids. PLoS Biol 6:e33

> Gettinby G, Robbins C, Lees F, Heuch PA, Finstad B, Malkenes R, Revie CW (2011) Use of a mathematical model to describe the epidemiology of Lepeophtheirus salmonis on farmed Atlantic salmon Salmo salar in the Hardangerfjord, Norway. Aquaculture 320:164-170

Heuch PA, Nordhagen JR, Schram TA (2000) Egg production in the salmon louse [Lepeophtheirus salmonis (Krøyer)] in relation to origin and water temperature. Aquacult Res 31:805-814

Jimenez DF, Revie CW, Hardy SP, Jansen PA, Gettinby G (2013) Multivariate evaluation of the effectiveness of treatment efficacy of cypermethrin against sea lice (Lepeophtheirus salmonis) in Atlantic salmon (Salmo salar). BMC Vet Res 9:258

Johnson SC, Albright LJ (1991) Development, growth, and survival of Lepeophtheirus salmonis (Copepoda: Caligidae) under laboratory conditions. J Mar Biol Assoc UK 71:425-436

> Jones PG, Hammell KL, Gettinby G, Revie CW (2013) Detection of emamectin benzoate tolerance emergence in different life stages of sea lice, Lepeophtheirus salmonis, on farmed Atlantic salmon, Salmo salar L. J Fish Dis 36: 209-220

> Kristoffersen AB, Rees EE, Stryhn H, Ibarra R, Campisto JL, Revie CW, St-Hilaire S (2013) Understanding sources of sea lice for salmon farms in Chile. Prev Vet Med 111: 165-175

> Kristoffersen AB, Jimenez D, Viljugrein H, Grøntvedt $\mathrm{R}$, Stien A, Jansen PA (2014) Large scale modelling of salmon lice (Lepeophtheirus salmonis) infection pressure based on lice monitoring data from Norwegian salmonid farms. Epidemics 9:31-39

Krkošek M, Bateman A, Proboszcz S, Orr C (2010) Dynamics of outbreak and control of salmon lice on two salmon farms in the Broughton Archipelago, British Columbia. Aquacult Environ Interact 1:137-146 
MCS (Marine Conservation Society) (2013) Cleaner fish position paper. www.mcsuk.org/downloads/fisheries/ Cleaner_Fish_Position_Paper.pdf (accessed 10 Oct 2014)

Middlemas SJ, Fryer RJ, Tulett D, Armstrong JD (2013) Relationship between sea lice levels on sea trout and fish farm activity in western Scotland. Fish Manag Ecol 20: 68-74

MSD Animal Health (2012) SLICE technical monograph. Merck Animal Health, Whitehouse Station, NJ

Proud R (2012) Dynamics of parasitic sea lice populations on salmon farms. Master's thesis, St. Andrews University

Revie CW, Robbins C, Gettinby G, Kelly L, Treasurer JW (2005) A mathematical model of the growth of sea lice, Lepeophtheirus salmonis, populations on farmed Atlantic salmon, Salmo salar L., in Scotland and its use in the assessment of treatment strategies. J Fish Dis 28:603-613

Rogers LA, Peacock SJ, McKenzie P, DeDominicis S and others (2013) Modeling parasite dynamics on farmed salmon for precautionary conservation management of wild salmon. PLoS ONE 8:e60096

Salama N, Rabe B (2013) Developing models for investigating the environmental transmission of disease-causing agents within open-cage salmon aquaculture. Aquacult

Editorial responsibility: Mark Costello,

Warkworth, New Zealand
Environ Interact 4:91-115

Skiftesvik AB, Bjelland RM, Durif CMF, Johansen IS, Browman HI (2013) Delousing of Atlantic salmon (Salmo salar) by cultured vs. wild ballan wrasse (Labrus bergylta). Aquaculture 402-403:113-118

SSPO (Scottish Salmon Producers Organisation) (2014) Fish health management report-July to September 2014. Scottish Salmon Producers Organisation, Perth

Stien A, Bjørn PA, Heuch PA, Elston DA (2005) Population dynamics of salmon lice Lepeophtheirus salmonis on Atlantic salmon and sea trout. Mar Ecol Prog Ser 290: 263-275

Stucchi DJ, Guo M, Foreman MGG, Czajko P, Galbraith M, Mackas DL, Gillibrand PA (2010) Modeling sea lice production and concentrations in the Broughton Archipelago, British Columbia. In: Jones S, Beamish R (eds) Salmon lice: an integrated approach to understanding parasite abundance and distribution. Wiley/Blackwell, Oxford, p 117-150

Tucker CS, Norman R, Shinn AP, Bron JE, Sommerville C, Wootten R (2002) A single cohort time delay model of the life-cycle of the salmon louse Lepeophtheirus salmonis on Atlantic salmon Salmo salar. Fish Pathol 37:107-118

Submitted: November 19, 2014; Accepted: April 14, 2015

Proofs received from author(s): May 5, 2015 